CERN-TH-6235/91

September 1991

\title{
MANIFESTLY $O(d, d)$ INVARIANT APPROACH TO SPACE-TIME DEPENDENT STRING VACUA
}

\author{
K.A. Meissner* and G. Veneziano \\ Theory Division, CERN
}

\begin{abstract}
An $O(d, d)$ symmetry of the manifold of string vacua that do not depend on $d$ (out of $D$ ) space-time coordinates has been recently identified. Here we write down, for $d=$ $D-1$, the low energy equations of motion and their general solution in a manifestly $O(d, d)$-invariant form, pointing out an amusing similarity with the renormalization group framework. Previously considered cosmological and black hole solutions are recovered as particular examples.
\end{abstract}

* Permanent address: Institute of Theoretical Physics, ul. Hoża 69, 00-681 Warszawa, Poland.

CERN-TH-6235/91

September 1991 


\section{INTRODUCTION}

Narain's original idea [1], [2] of an $O(d, d)$ symmetry acting on the manifold of static, $d$-dimensional string compactifications, has been recently generalized [3] to the case of massless backgrounds (metric, torsion and dilaton) which simply do not depend upon a particular set of (possibly non-compact) string coordinates $X^{a}(a=1,2, \ldots d)$.

A $\sigma$-model argument for the validity of such a symmetry at all orders in $\alpha^{\prime}$ (and possibly at higher genus as well) runs as follows [3]: Consider a conformal background (a string vacuum) of the kind specified above: the associated nilpotent BRST operator $Q$ will depend trivially (i.e. quadratically at most) on the $2 d$ phase-space variables $Z=$ $\left(P_{a}, X^{\prime a}\right)$. Perform now [3] a global, canonical $O(d, d)$ transformation on the $Z s$. Since this transformation preserves commutation relations and Wick contractions, the new BRST operator will also be nilpotent. However, as it turns out, the change in $Z$ can be traded for a change in the backgrounds, implying that also the transformed backgrounds define a (generally inequivalent) conformal theory. $O(d, d)$ is thus a symmetry of this particular class of string vacua.

Depending on the theory at hand, a subgroup of $O(d, d)$, which we shall call the - gauge group- $\mathcal{G}$, leaves the theory unchanged. Inequivalent vacua are thus points in the coset $O(d, d) / \mathcal{G}$. While for the static, fully compact case one has [1], [2] the $d^{2}$-dimensional space given by $\mathcal{G}=O(d) \otimes O(d)$, for the completely non-compact (but time-dependent) case the coset has dimensionality $d(d-1) / 2$ [3]. It has been argued by Sen [4] that, in this latter case, our coset is equivalent to $O(d) \otimes O(d) / O(d)$ and that the $O(d) \otimes O(d)$ symmetry persists to all orders in $\alpha^{\prime}$. For the sake of generality we shall base our discussion here on the full original $O(d, d)$ symmetry.

Discrete subgroups of $O(d, d)$ (somewhat improperly called - duality transformations-) had been previously discussed both in the context of cosmological solutions [5], [6] and in that of $2 D$ black-holes [7], [8], [9]. Finally, the complete $O(d, d)$ group has been employed to generate, from some known solutions, radically new candidate conformal backgrounds both in the black-hole [10] and in the cosmological [11] case. An interesting aspect of $O(d, d)$, made apparent by these constructions, is that it can transform gauge-equivalent backgrounds into gauge-inequivalent ones, as evidentiated by the presence of a non trivial torsion field $B$ in the transformed solutions. 
In this paper we shall continue our work [3] by rewriting, for $d=D-1$, the field equations in a manifestly $O(d, d)$-invariant form. This allows us to give the general solution of the (lowest order) equations in terms of quadratures. Actually, equations (and solutions) take the form of renormalization-group equations (and solutions) in real cosmic time both for the coupling constant of string theory and for the matrix descibing the metric and torsion backgrounds. This analogy is used in order to obtain a general classification of the solutions. Finally, we shall recover several known backgrounds as particular cases of our general solution.

\section{2. $O(d, d)$ SYMMETRIC EQUATIONS OF MOTION AND THEIR GENERAL SOLUTION}

In this Section we shall derive manifestly $O(d, d)$-invariant equations of motion for the low energy effective action of string gravity coupled to the dilaton and the antisymmetric tensor:

$$
S=\int d^{D} x \sqrt{-G} e^{-\phi}\left[\Lambda-R-G^{\mu \nu} \partial_{\mu} \phi \partial_{\nu} \phi-\frac{1}{12} H_{\mu \nu \rho} H^{\mu \nu \rho}\right]
$$

Here $\Lambda$ is the cosmological constant (proportional to D-10), $\phi$ is the (Fradkin-Tseytlin) dilaton [12] $G_{\mu \nu}$ is the $\sigma$-model metric and $H_{\mu \nu \rho}$ is expressed, as usual, in terms of $B_{\mu \nu}$ via :

$$
H_{\mu \nu \rho}=\partial_{\mu} B_{\nu \rho}+\text { cyclic }
$$

If $G$ and $B$ are only functions of time, invariance under general coordinate transformations and under $B_{\mu \nu} \rightarrow B_{\mu \nu}++\partial_{[\mu} \Lambda_{\nu]}$ allows always to bring them to the form:

$$
G=\left(\begin{array}{cc}
-1 & 0 \\
0 & G(t)
\end{array}\right), \quad B=\left(\begin{array}{cc}
0 & 0 \\
0 & B(t)
\end{array}\right)
$$

Hereafter we shall use the symbols $G$ and $B$ to denote the $d \times d$ matrices appearing in (3).

As shown in [3], by introducing a $2 d \times 2 d$ matrix $M$ and a field $\Phi$ defined as

$$
\begin{gathered}
M=\left(\begin{array}{cc}
G^{-1} & -G^{-1} B \\
B G^{-1} & G-B G^{-1} B
\end{array}\right) \\
\Phi=\phi-\ln \sqrt{\operatorname{det} G} .
\end{gathered}
$$


the action (1) takes the form:

$$
S=\int d t e^{-\Phi}\left\{\Lambda+(\dot{\Phi})^{2}+\frac{1}{8} \operatorname{Tr}[\dot{M} \eta \dot{M} \eta]\right\}
$$

where $\eta$ defines an $O(d, d)$ metric in off-diagonal form i.e.

$$
\eta=\left(\begin{array}{ll}
0 & 1 \\
1 & 0
\end{array}\right)
$$

The action (6) is manifestly invariant under the a global $O(d, d)$ group acting as :

$$
\begin{gathered}
\Phi \rightarrow \Phi, \quad M \rightarrow \Omega^{T} M \Omega \\
\Omega^{T} \eta \Omega=\eta
\end{gathered}
$$

For $d<D-1$ and backgrounds $G$ and $B$ which are not block-diagonal, the $\sigma$-model argument given in Sect.1 still suggests an $O(d, d)$ symmetry with the off-diagonal blocks of $M$ transforming s by the obvious left or right multiplication with $\Omega$.

We will now obtain manifestly $O(d, d)$-invariant equations of motion from the action (6). The first equation follows from reintroducing $G_{00}$ in the action and from setting to zero the corresponding variation. This gives directly the "Zero Energy" condition

$$
(\dot{\Phi})^{2}+\frac{1}{8} \operatorname{Tr}[\dot{M} \eta \dot{M} \eta]-V=0
$$

where we allow now for a more general potential $V(\Phi)$ rather than just a constant. If we assume that the potential does not break the symmetry explicitly then it can depend on $M$ only through a function of the invariants $\operatorname{Tr}(M \eta)^{p}, p=1,2, \ldots$. However, for $p$ odd these traces vanish and for $p$ even they are equal to $2 d$; hence the potential can depend only on $\Phi$.

The variation of the action with respect to $\Phi$ yields:

$$
(\dot{\Phi})^{2}-2 \ddot{\Phi}-\frac{1}{8} \operatorname{Tr}[\dot{M} \eta \dot{M} \eta]+\frac{\partial V}{\partial \Phi}-V=0
$$

The variation of the action with respect to $M$ has to be done carefully, since $M$ is subject to several constraints. The simplest way to proceed is to write

$$
\delta M=\Omega^{T} M \Omega-M
$$


where $\Omega=1+\epsilon$ belongs to $O(d, d)$. Expanding the variation of the action to terms linear in $\epsilon$ and using the fact that $\epsilon \eta$ is antisymmetric we get the equation:

$$
\partial_{t}(M \eta \dot{M})=\dot{\Phi}(M \eta \dot{M})
$$

which can be immediately integrated once to give:

$$
\mathrm{e}^{-\Phi}(M \eta \dot{M})=\mathrm{const}=A
$$

From its definition the constant matrix $A$ satisfies

$$
A^{T}=-A, \quad M \eta A=-A \eta M .
$$

It is obvious that eqs. (10), (11) and (13) are invariant under the full $O(d, d)$ group defined by (8) and (9).

Substituting (14) into (10) we obtain the first order equation for $\Phi$ :

$$
(\dot{\Phi})^{2}=\frac{\exp (2 \Phi)}{8} \operatorname{Tr}(A \eta)^{2}+V(\Phi)
$$

which can be solved by quadratures:

$$
t=\int_{\Phi_{0}}^{\Phi} d y\left(\frac{\exp (2 y)}{8} \operatorname{Tr}(A \eta)^{2}+V(y)\right)^{-1 / 2}
$$

It can be easily shown that the solution of (16) automatically satisfies (11), provided that $\dot{\Phi} \neq 0$. The solution (17) can be used next to define a "dilaton time" $\tau$ :

$$
\tau=\int_{t_{0}}^{t} \mathrm{e}^{\Phi} d t^{\prime}
$$

In terms of $\tau$ the general solution of (14) simply reads :

$$
M(t)=\exp (-A \eta \tau) M\left(t_{0}\right)
$$

Hence the whole solution can be explicitly given in terms of quadratures, which is a welcome surprise for a complicated system such as the one described by (1).

To end this Section we would like to point out an amusing similarity between equations (16), (18) and (19) and the renormalization group (RG) equations. Introducing the "reduced" coupling constant $\alpha$ by:

$$
\alpha=\mathrm{e}^{\Phi}
$$


we can rewrite (16) as a RG-equation for the running of $\alpha$ :

$$
\frac{d \alpha}{d t}=\beta(\alpha)
$$

where the $\beta$-function is given by:

$$
\beta(\alpha)= \pm \alpha \sqrt{V(\alpha)+\frac{\alpha^{2}}{8} \operatorname{Tr}(A \eta)^{2}}
$$

Once eq. (22) is solved, eq. (18) is the usual definition of the "RG-time" in terms of which moments of structure functions evolve to leading order exponentially (generally with a matrix-valued exponent because of operator mixing), i.e. precisely as in eq. (19). The standard RG analysis of UV or IR fixed points can be applied to our system choosing, for definiteness, the plus sign in eq. (22) (this corresponds, physically, to choosing solutions which evolve from weak coupling in the far past). This analysis leads to the following classification scheme for the general solution:

a) If $\beta(\alpha)$ has no other zero but the trivial one then we have two possibilities:

$\left.\mathrm{a}_{1}\right)$

$$
\int_{\alpha_{0}}^{\infty} \frac{d x}{\beta(x)}<\infty
$$

There is a singularity in $\Phi$ at a finite $t=t_{c}$. There can also be a (coordinate or curvature) singularity if

$$
\int_{\alpha_{0}}^{\infty} \frac{d x x}{\beta(x)}=\infty
$$

In this case the typical cosmology near $t_{c}$ is superinflationary (Cf. [5]).

$\left.\mathrm{a}_{2}\right)$

$$
\int_{\alpha_{0}}^{\infty} \frac{d x}{\beta(x)}=\infty
$$

In this case no singularity can develop at any finite $t$.

b) If $\beta(\alpha)$ has a zero at some $\alpha^{*}>0$ then one has again two possibilities:

$\left.\mathrm{b}_{1}\right)$

$$
\int_{\alpha_{0}}^{\alpha^{*}} \frac{d x}{\beta(x)}<\infty
$$

No singularity develops at any finite $t$ and the coupling constant, after growing to $\alpha^{*}$, goes back to zero after an infinite time.

$\left.\mathrm{b}_{2}\right)$

$$
\int_{\alpha_{0}}^{\alpha^{*}} \frac{d x}{\beta(x)}=\infty
$$


In this case the theory has a genuine late-time fixed point $\alpha=\alpha^{*}$. At late times a regime of exponential inflation $\left(M(t) \sim \exp \left(\alpha^{*} A \eta t\right)\right)$ sets in.

\section{COSMOLOGICAL SOLUTIONS AND 2D BLACK HOLES AS PAR-} TICULAR CASES

In this Section we will solve the equations (16) and (19) for some special potentials. The solutions we find include time dependent cosmological solutions of [5] and 2D black holes [7], [8], as well as some recently studied "boosted" versions of both [11], [10], but the family of solutions of (16) and (19) is much larger.

We start with the case of vanishing potential $V=0$ (critical dimensions). In this case (16) can be immediately solved to give

$$
\mathrm{e}^{\Phi}=\alpha=C /(T-t) ; \quad C=\sqrt{8 / \operatorname{Tr}(A \eta)^{2}}
$$

From (18) we get

$$
\tau=C \ln \frac{T-t}{T-t_{0}}
$$

so that the solution for $M$ reads:

$$
M(t)=\exp \left(C A \eta \ln \frac{T-t}{T-t_{0}}\right)
$$

where we assumed that $M\left(t_{0}\right)=\mathbf{1}$.

To be more explicit let us assume some specific form for $A$ :

$$
A=\left(\begin{array}{cc}
0 & -A_{d} \\
A_{d} & 0
\end{array}\right)
$$

where $A_{d}=\operatorname{diag}\left(a_{1}, . ., a_{d}\right)$. In this case (30) reads:

$$
M(t)=\left(\begin{array}{cc}
\operatorname{diag}\left[\left(\frac{T-t}{T-t_{0}}\right)^{-2 \alpha_{1}}, . .\right] & 0 \\
0 & \operatorname{diag}\left[\left(\frac{T-t}{T-t_{0}}\right)^{2 \alpha_{1}}, . .\right.
\end{array}\right)
$$

where $\alpha_{i}=a_{i} / \sqrt{\Sigma a_{i}^{2}}$. These solutions are exactly the ones discussed in [5], [6] (see also [13). For $\alpha_{i}=\alpha \delta_{i 1}$ we recover the special case of the completely flat "Milne" metric 
[11], while, by inverting and boosting the corresponding $A$, we arrive at the one-parameter family of non-trivial backgrounds discussed in [1]].

We now want to discuss the solutions in presence of the potential $V=\Lambda=$ const. The solution of (16) can be immediately given:

$$
\mathrm{e}^{\Phi}=\alpha=C \sqrt{\Lambda} / \sinh (\sqrt{\Lambda}(T-t)) ; \quad C=\sqrt{8 / \operatorname{Tr}(A \eta)^{2}}
$$

From (18) we get

$$
\tau=C \ln \frac{\tanh (\sqrt{\Lambda}(T-t) / 2)}{\tanh \left(\sqrt{\Lambda}\left(T-t_{0}\right) / 2\right)}
$$

so that the solution for $M$ reads:

$$
M(t)=\exp \left(C A \eta \ln \frac{\tanh (\sqrt{\Lambda}(T-t) / 2)}{\tanh \left(\sqrt{\Lambda}\left(T-t_{0}\right) / 2\right)}\right)
$$

where we again assumed that $M\left(t_{0}\right)=\mathbf{1}$.

For $d=1$ we take

$$
A=\left(\begin{array}{cc}
0 & -a \\
a & 0
\end{array}\right) \quad a>0
$$

and assume that $M(-\infty)=\mathbf{1}$. Then

$$
\begin{gathered}
M(t)=\left(\begin{array}{cc}
\tanh ^{-2}(\sqrt{\Lambda}(T-t) / 2) & 0 \\
0 & \tanh ^{2}(\sqrt{\Lambda}(T-t) / 2)
\end{array}\right) \\
\mathrm{e}^{\Phi}=\frac{2 \sqrt{\Lambda}}{a \sinh (\sqrt{\Lambda}(T-t))}
\end{gathered}
$$

After the identification [3]:

$$
t \leftrightarrow i x, T=0, \Lambda=-4
$$

we recover the $k=9 / 4$ black hole solution of [7], [8]. Acting on the solution with $\eta$ we recover the "dual" $2 \mathrm{D}$ black hole of ref. [9].

For $d>1$ we can use the same $A$ (with zeroes in the additional entries) and reproduce the $k \neq 9 / 4$ black holes of [7], while, by boosting any of these solutions one recovers the "twisted" black holes of [10].

We wish to conclude by mentioning one amusing solution that exists for $d=9$ (usual uncompactified superstring). As $A$ we take

$$
A=\left(\begin{array}{cc}
0 & \operatorname{diag}\left(-a_{1}, . .,-a_{9}\right) \\
\operatorname{diag}\left(a_{1}, . ., a_{9}\right) & 0
\end{array}\right)
$$


Then $M$ is equal to

$$
M=\left(\begin{array}{cc}
\operatorname{diag}\left(\tanh ^{-2 \alpha_{1}}(\sqrt{\Lambda}(T-t) / 2), . .\right) & 0 \\
0 & \operatorname{diag}\left(\tanh ^{2 \alpha_{1}}(\sqrt{\Lambda}(T-t) / 2), . .\right)
\end{array}\right)
$$

where $\alpha_{i}=a_{i} / \sqrt{\Sigma a_{i}^{2}}$. The scalar curvature and dilaton are given by:

$$
\begin{gathered}
R=-\frac{\Lambda}{\left.\cosh ^{2}(\sqrt{\Lambda}(T-t)) / 2\right)}\left[\sum \alpha_{i}-\frac{\left(\Sigma \alpha_{i}-1\right)^{2}}{\left.4 \sinh ^{2}(\sqrt{\Lambda}(T-t) / 2)\right)}\right] \\
\mathrm{e}^{\phi}=\frac{(\tanh (\sqrt{\Lambda}(T-t) / 2))^{\Sigma \alpha_{i}-1}}{\left.a \cosh ^{2}(\sqrt{\Lambda}(T-t) / 2)\right)} .
\end{gathered}
$$

Hence we have a singularity for $t \rightarrow T$ unless

$$
\sum \alpha_{i}=1
$$

Assuming that all $\left|\alpha_{i}\right|$ are equal, the only solution for $\alpha_{i}$ is $(-1 / 3,-1 / 3,-1 / 3,+1 / 3, . .,+1 / 3)$ so that three dimensions expand and six contract!

We are grateful to D. Amati, M. Gasperini and E. Rabinovici for discussions. 


\section{References}

[1] K. S. Narain, Phys. Lett. B169 (1986) 41.

[2] K. S. Narain, M. H. Sarmadi and E. Witten, Nucl. Phys. B279 (1987) 369.

[3] K.A. Meissner and G. Veneziano, Symmetries of Cosmological Superstring Vacua, CERN-TH.6138/91, to appear in Phys.Lett.B.

[4] A. Sen, $O(d) \otimes O(d)$ Symmetry of the Space of Cosmological Solutions in String Theory, Scale Factor Duality, and Two Dimensional Black Holes, Tata Institute preprint, TIFR/TH/91-35.

[5] G.Veneziano, Scale Factor Duality for Classical and Quantum Strings, CERN-TH$6077 / 91$.

[6] A. A. Tseytlin, Duality and Dilaton, Mod. Phys. Lett. 6A (1991) 1721; Space-Time Duality, Dilaton and String Cosmology, to appear in the Proceedings of the First International A. D. Sakharov's Conference in Physics, Moscow, May 1991.

[7] E.Witten, On String Theory and Black Holes, IASSNS-HEP-91/12.

[8] G. Mandal, A.M. Sengupta and S.R. Wadia, Classical Solutions of 2-Dimensional String Theory, IASSNS-HEP-91/10; see also, K. Bardakci, A. Forge and E. Rabinovici, Nucl. Phys. B344 (1990) 344; S. Elitzur, A. Forge and E. Rabinovici, Nucl. Phys. B359 (1991) 581; I. Bars and D. Nemeschansky, Nucl. Phys. B348 (1991) 89.

[9] A. Giveon, Target Space Duality and Stringy Black Holes, LBL-30671; E. Smith and J. Polchinski, preprint, UTTG-07-91; R. Dijkgraaf, H. Verlinde and E. Verlinde, preprint LBL-30747 (1991).

[10] A. Sen, Twisted Black p-brane Solutions in String Theory, Tata Institute preprint, TIFR/TH/91-37.

[11] M. Gasperini, J. Maharana and G. Veneziano, From Trivial to Non-Trivial Conformal String Backgrounds via $O(d, d)$ Transformations,preprint CERN-TH-6214/91

[12] E. S. Fradkin and A. A. Tseytlin, Phys. Lett. 158B (1985) 316; E. Witten, Phys. Lett. 149B (1984) 351.

[13] M. Mueller, Nucl. Phys. B337 (1990) 37. 